Published in final edited form as:

Physiol Genomics. 2008 February 19; 32(3): 401-408. doi:10.1152/physiolgenomics.00241.2007.

\title{
Quantitative trait loci for physical activity traits in mice
}

\author{
J. Timothy Lightfoot ${ }^{1}$, Michael J. Turner ${ }^{1}$, Daniel Pomp ${ }^{3}$, Steven R. Kleeberger ${ }^{4}$, and Larry \\ J. Leamy ${ }^{2}$ \\ ${ }^{1}$ Department of Kinesiology, University of North Carolina, Charlotte \\ ${ }^{2}$ Department of Biology, University of North Carolina, Charlotte \\ ${ }^{3}$ Departments of Nutrition and Cell and Molecular Physiology and Carolina Center for Genome \\ Science, University of North Carolina, Chapel Hill \\ ${ }^{4}$ Laboratory of Respiratory Biology, National Institute of Environmental Health Sciences, Durham, \\ North Carolina
}

\section{Abstract}

The genomic locations and identities of the genes that regulate voluntary physical activity are presently unknown. The purpose of this study was to search for quantitative trait loci (QTL) that are linked with daily mouse running wheel distance, duration, and speed of exercise. $\mathrm{F}_{2}$ animals $(n=$ 310 ) derived from high active $\mathrm{C} 57 \mathrm{~L} / \mathrm{J}$ and low active $\mathrm{C} 3 \mathrm{H} / \mathrm{HeJ}$ inbred strains were phenotyped for 21 days. After phenotyping, genotyping with a fully informative single-nucleotide polymorphism panel with an average intermarker interval of $13.7 \mathrm{cM}$ was used. On all three activity indexes, sex and strain were significant factors, with the $\mathrm{F}_{2}$ animals similar to the high active $\mathrm{C} 57 \mathrm{~L} / \mathrm{J}$ mice in both daily exercise distance and duration of exercise. In the $\mathrm{F}_{2}$ cohort, female mice ran significantly farther, longer, and faster than male mice. QTL analysis revealed no sex-specific QTL but at the 5\% experimentwise significance level did identify one QTL for duration, one QTL for distance, and two QTL for speed. The QTL for duration (DUR13.1) and distance (DIST13.1) colocalized with the QTL for speed (SPD13.1). Each of these QTL accounted for $~ 6 \%$ of the phenotypic variance, whereas SPD9.1 (chromosome 9, $7 \mathrm{cM}$ ) accounted for $11.3 \%$ of the phenotypic variation. DUR13.1,

DIST13.1, SPD13.1, and SPD9.1 were subsequently replicated by haplotype association mapping. The results of this study suggest a genetic basis of voluntary activity in mice and provide a foundation for future candidate gene studies.

\section{Keywords}

genetics; locomotion; linkage mapping

THERE IS SUBSTANTIAL EVIDENCE that physical inactivity contributes to a large variety of chronic health conditions (e.g., Refs. 3,28). Several studies with animals and humans have suggested that genetic factors may play a significant role in regulating voluntary daily activity $(12,16,19,31$,

Copyright () 2005 by the American Physiological Society.

Address for reprint requests and other correspondence: J. T. Lightfoot, Dept. of Kinesiology, UNC Charlotte, 9201 University City Blvd., Charlotte, NC 28223 (e-mail: tlightfoot@uncc.edu)..

GRANTS

This project was supported by National Institutes of Health Grants DK-61635 (J. T. Lightfoot), AR-050085 (J. T. Lightfoot, M. J. Turner, L. J. Leamy), AG-022417 (M. J. Turner), and DK-076050 (D. Pomp) and intramural funding from the National Institute of Environmental Health Sciences (S. R. Kleeberger). 
35). However, no investigations have reported the location or identity of the genetic factors involved in regulating voluntary physical activity.

Previous attempts at identifying locomotor-related behavior genetic linkage have provided tantalizing suggestions regarding possible chromosomal locations of regulating genes. Conti et al. (5) identified two significant quantitative trait loci (QTL) related to open-maze locomotion behavior, Gershenfeld and colleagues (8) identified several significant QTL related to open-field activity, and Toth and Williams (33) identified several suggestive QTL linked to the amplitude of the oscillation in daily activity in mice. Recent data have suggested that shortterm behavioral locomotor tests, such as maze locomotion and open-field activity, are more tests of fear and anxiety and do not correlate well with home cage activity $(23,24)$. Therefore, previously identified QTL associated with short-term locomotor behaviors may not be associated with activity that is truly spontaneous and voluntary.

We therefore initiated a study to search for QTL that are associated with how far (distance), how long (duration), and how fast (speed) mice run on a running wheel permanently mounted in their home cage. This study involved a full genome scan for QTL for these traits in the $F_{2}$ descendants of two inbred strains that were previously shown (19) to exhibit significant differences in daily physical activity (C57L/J, high active; $\mathrm{C} 3 \mathrm{H} / \mathrm{HeJ}$, low active). Several QTL were discovered for each of the activity traits in this population of mice, and their individual additive and dominance effects on the traits were estimated. We also replicated the existence and refined the genomic position of the two QTL with the largest effect on activity, using haplotype association mapping (HAM) in 27 inbred mouse strains.

\section{METHODS}

\section{Animals}

The University of North Carolina Charlotte Institutional Animal Care and Use Committee approved the procedures in this study. We followed the guidelines for ethical use of animals from the American Physiological Society and the American College of Sports Medicine.

In a previous study (19) we identified $\mathrm{C} 57 \mathrm{~L} / \mathrm{J}$ inbred mice (generation $\mathrm{F}_{130}$ ) as having high daily physical activity and $\mathrm{C} 3 \mathrm{H} / \mathrm{HeJ}$ inbred mice (generation $\mathrm{F}_{170}$ ) as having low daily physical activity. Ten C57L/J mice ( 5 males and 5 females) and $10 \mathrm{C} 3 \mathrm{H} / \mathrm{HeJ}$ mice $(5$ females and 5 males) underwent a reciprocal breeding protocol that produced $63 \mathrm{~F}_{1}$ mice: $32 \mathrm{C} 3 \mathrm{C} 57 \mathrm{~F}_{1 \mathrm{a}}$ mice from $\mathrm{C} 3 \mathrm{H} / \mathrm{HeJ}$ mothers and $\mathrm{C} 57 \mathrm{~L} / \mathrm{J}$ fathers and $31 \mathrm{C} 57 \mathrm{C} 3 \mathrm{~F}_{1 \mathrm{~b}}$ mice from $\mathrm{C} 57 \mathrm{~L} / \mathrm{J}$ mothers and $\mathrm{C} 3 \mathrm{H} / \mathrm{HeJ}$ fathers. The $\mathrm{F}_{1}$ mice were weaned at $21-28$ days and phenotyped for voluntary daily activity (see below), and then $10 \mathrm{C} 57 \mathrm{C} 3 \mathrm{~F}_{1 \mathrm{~b}}$ ( 5 males and 5 females) and $10 \mathrm{C} 3 \mathrm{C} 57 \mathrm{~F}_{1 \mathrm{a}}$ (5 males and 5 females) mice were randomly chosen for reciprocal crossing.

An attempt was made to produce at least $300 \mathrm{~F}_{2}$ offspring from these $\mathrm{F}_{1}$ matings. This sample size was expected to be a reasonable compromise between the labor-intensive difficulties associated with the phenotyping process (see below) and the achievement of an acceptable level of statistical power for the detection of QTL (18). With a sample size of 300, the statistical power to detect a QTL that contributes $5 \%$ of the total variation in a trait is 0.98 if its effects are entirely additive or 0.90 if there is complete dominance (22). For a QTL contributing only $2.5 \%$, the statistical power is still relatively high: $78 \%$ if there is no dominance and $61 \%$ for complete dominance (22). Although it is known that the effects of QTL detected with very low statistical power may be considerably overestimated, those estimated with $60-98 \%$ statistical power have very little inflation (1).

The $\mathrm{F}_{1}$ crosses were successful in producing a total of $310 \mathrm{~F}_{2}$ progeny. There were four resulting types of $F_{2}$ offspring (i.e., $F_{2}$ substrains): $F_{2 v}=F_{2}$ mice that had $C 57 C 3 F_{1 b}$ mothers and $C 3 C 57$ 
$\mathrm{F}_{1 \mathrm{a}}$ fathers $(\mathrm{C} 57 \mathrm{C} 3 / \mathrm{C} 3 \mathrm{C} 57 ; n=100) ; \mathrm{F}_{2 \mathrm{w}}=\mathrm{F}_{2}$ mice that had $\mathrm{C} 3 \mathrm{C} 57 / \mathrm{C} 57 \mathrm{C} 3$ progenitors $(n=$ 84); $\mathrm{F}_{2 \mathrm{x}}=\mathrm{F}_{2}$ mice that had C3C57/C3C57 progenitors $(n=71)$; and $\mathrm{F}_{2 \mathrm{y}}=\mathrm{F}_{2}$ mice that had $\mathrm{C} 57 \mathrm{C} 3 / \mathrm{C} 57 \mathrm{C} 3$ progenitors $(n=55)$. The $\mathrm{F}_{2}$ mice of all four types were weaned at 21-28 days and phenotyped. All mice were housed in the same room in the University Vivarium, which was maintained at $18-21^{\circ} \mathrm{C}$ and $20-40 \%$ humidity with $12: 12$-h light-dark cycles. Food (Harland Teklad 8604 Rodent Diet, Madison, WI) and water were provided ad libitum. Each mouse was weighed to the nearest $0.1 \mathrm{~g}$ weekly.

\section{Phenotyping Daily Wheel Running Activity Level}

Phenotyping of daily activity was conducted as in past studies from our lab $(19,35)$. Briefly, after weaning mice were housed individually in a rat cage with a solid-surface running wheel mounted in the cage (145-mm diameter, Ware Manufacturing, Phoenix, AZ). A magnetic sensor was glued to the running wheel that was interfaced with a small bicycle computer (either BC600 or BC500, Sigma Sport, Olney, IL) that counted the total wheel revolutions and time each mouse spent exercising (19). Total daily distance $(\mathrm{km})$ and total daily exercise time (min) were recorded every $24 \mathrm{~h}$ for each mouse, with average daily running speed $(\mathrm{m} / \mathrm{min})$ being subsequently calculated by dividing distance by duration. For the $F_{1}$ and $F_{2}$ cohorts, these three physical activity level phenotypes were measured continuously for 21 days beginning when the mice were 63 days old (9 wk) to account for any fluctuations in daily wheel running due to menstrual cycling in the female mice and to prevent confounding due to the mice learning to run on the wheels. The male parental mice were also phenotyped between 9 and 11 wk of age. Because of time constraints, the female parental mice were not phenotyped, but representative female data from earlier strain screens (19) were used for phenotypic comparisons. During phenotyping, each mouse used the same wheel throughout the 21-day monitoring period, and the wheels were checked on a daily basis to ensure that they turned freely.

\section{Genotyping}

Within 7 days after the phenotyping was completed the mice were anesthetized with 2-4\% inhaled isofluorine. Kidneys were then harvested, flash frozen in liquid nitrogen, and stored at $-80^{\circ} \mathrm{C}$ for later genotyping. DNA extraction from kidneys was performed as previously described (21) with a DNeasy kit (Qiagen, Valencia, CA). Genotyping of 129 single-nucleotide polymorphisms (SNPs) was conducted by GeneSeek (Lincoln, NE) with a matrix-assisted laser desorption ionization-time of flight mass spectrometry (MALDI-TOF MS) system. All SNPs chosen were polymorphic between the progenitor strains (Wellcome-CTC Mouse Strain SNP Genotype Set; Ref. 36) and covered the entire mouse genome with an average intermarker interval of $13.7 \mathrm{cM}$. Results are reported with NCBI Build 36.1 SNP locations.

\section{Statistics}

General linear modeling (GLM, JMP 5.1, SAS Institute, Cary, NC) was used to test for the contribution of strain (i.e., $\mathrm{C} 57 \mathrm{~L} / \mathrm{J}, \mathrm{C} 3 \mathrm{H} / \mathrm{HeJ}, \mathrm{F}_{1}, \mathrm{~F}_{2}$ ), substrain group within the $\mathrm{F}_{2}$ cohort, sex, weight, and all appropriate interactions on each activity phenotype (i.e., daily distance, duration, and exercise speed). Alpha values were set a priori at 0.05 , variables that did not contribute significantly to the models were dropped, and the analysis was repeated with standard ANOVA methods. To determine pairwise differences (e.g., between sexes and strains) in each trait, subsequent post hoc testing was completed with Tukey-Kramer honestly significant difference (HSD). Broad-sense heritability was calculated from the $\mathrm{F}_{2}$ cohort with the degree of genetic determination $\left(\mathrm{d}^{2}\right)$, which was estimated by the formula $\mathrm{d}^{2}=\left(\mathrm{V}_{\mathrm{F} 2}-\right.$ $\left.\mathrm{V}_{\mathrm{F} 1}\right) / \mathrm{V}_{\mathrm{F} 2}$, where $\mathrm{V}_{\mathrm{F} 1}$ and $\mathrm{V}_{\mathrm{F} 2}$ are the variances of the $\mathrm{F}_{1}$ and $\mathrm{F}_{2}$ populations, respectively. The standard error of the degree of genetic determination was estimated with the formula 
$s_{d}^{2}=\left(1-\mathrm{d}^{2}\right)\left[2\left(1 / \mathrm{N}_{1}+1 / \mathrm{N}_{2}\right)\right]^{1 / 2}$, where $\mathrm{N}_{1}$ and $\mathrm{N}_{2}$ are the degrees of freedom in the $\mathrm{F}_{1}$ and $\mathrm{F}_{2}$ populations, respectively (7).

Preliminary statistical adjustment-Before the QTL analyses, we first tested the three activity traits (i.e., distance, duration, speed) for potential differences due to sex, litter size, rearing block, and body weight effects. These tests were accomplished in analyses of covariance in which sex, litter size, and block effects were treated as classification factors and body weight was treated as a covariate. If any of these factors were significantly associated with any of the activity traits, we adjusted the data for these nongenetic effects. Normality tests were then conducted on the data to determine the need for subsequent transformation to normalize the data.

Experimental QTL determination-Separate QTL analyses were run for the three traits with the interval mapping model (10) with a canonical correlation approach that we described previously (15). To implement this approach, additive genotypic index values of $-1,0$, and +1 and dominance genotypic index values of $-0.5,+0.5$, and -0.5 were assigned for $\mathrm{C} 3 \mathrm{H} / \mathrm{HeJ}$ homozygotes, heterozygotes, and C57L/J homozygotes, respectively, at the site of each SNP marker. We also imputed genotypic index values for all locations $2 \mathrm{cM}$ apart between flanking SNP markers on each chromosome, using the recombination percentages given in the Mouse Genome Database (6) and the equations of Haley and Knott (10). The canonical analyses generated $F$ values with their associated probabilities that were converted to a linear scale by logarithmic transformation $\left[\mathrm{LPR}=\log _{10}(1 /\right.$ Prob) $]$ to make the results comparable to logarithm of odds (LOD) scores obtained via maximum likelihood analysis (13). If the highest LPR calculated for a given chromosome exceeded a specific threshold value, a QTL was considered to be present at the position associated with that LPR score.

The effective marker number approach of Cheverud (4) was used to generate specific threshold values for each chromosome (chromosomewise 5\% values) as well as an overall 5\% experimentwise threshold value. This approach does not require permutation runs but instead calculates $5 \%$ chromosomewise threshold values by division of 0.05 by the number of effective markers on each chromosome (obtained from the variance of the eigenvalues of intermarker correlations) and the $5 \%$ experimentwise threshold value by dividing 0.05 by the sum of the effective number of markers over all chromosomes (4). The 5\% experimentwise specific threshold value for our $\mathrm{F}_{2}$ mouse population was determined to be equal to 3.39 (i.e., significant LPR value $=3.39$ ), while the $5 \%$ chromosomewise specific threshold values ranged from 1.77 to 2.13 with a mean of 1.97. LPR scores exceeding their appropriate chromosomewise threshold value were considered suggestive of linkage, whereas those exceeding the experimentwise threshold value were taken as significant evidence of linkage. Confidence intervals for each QTL were determined by the one-LOD rule (22).

Each chromosome was also tested for two-QTL and sex-specific QTL effects. Sex-specific effects were tested by first assigning a code for the sex of each individual. We than ran a whole genome scan for each trait and tested for the interaction of sex with the additive and dominance genotypic index values (partialling the main effects due to genotypic values and sex). Any interactions exceeding their appropriate chromosomewise threshold values were taken to represent QTL whose effects differed in the two sexes. In those instances where the interactions exceeded the threshold values, the QTL analyses were repeated for the separate sexes. Tests for two QTL on a given chromosome were done by comparing the $\chi^{2}$ values generated for the one- and two-QTL models. Where two QTL on a given chromosome were indicated, confidence intervals for each QTL were obtained as before, but partialling out the effects of the other QTL on that chromosome. 
Multiple regressions of each trait on the additive and dominance genotypic index values were run for all QTL affecting that trait to estimate additive ( $a$ ) and dominance $(d)$ genotypic values and to test them for significance (using individual $t$-tests). The $a$ values estimate one-half of the difference between the average phenotypic values of the two homozygotes, and the $d$ values estimate the difference between the average phenotypic value of the heterozygotes and the midpoint between the two homozygote genotypic values. These values are therefore useful in expressing the magnitude of effect of the QTL and were standardized (divided by the standard deviation) to permit comparisons of these effects among the three traits.

Epistasis analysis-Once QTL were discovered for each of the three activity traits, significant interactions (epistasis) among each pair of QTL on different chromosomes were investigated. We expected to be able to detect any epistasis present because significant epistatic interactions among QTL for various traits have been discovered in other mouse populations of similar size (see, e.g., Ref. 37). Epistasis determination was accomplished by multiple regression of each trait on the additive and dominance genotypic index values for each of two QTL and the four interactions of the additive and dominance values. Tests for the overall significance of epistasis for each combination of QTL were made with an $F$ statistic (numerator $\mathrm{df}=4$ ) generated from testing the four interaction terms. We used the conventional 5\% significance level without adjustment for multiple comparison problems since the locations for epistasis testing were chosen without any prior knowledge of potential epistatic effects (9). The regression coefficients associated with these four interactions estimated additive by additive ( $a a$ ), additive by dominance ( $a d)$, dominance by additive $(d a)$, and dominance by dominance $(d d)$ genotypic epistatic terms. For those pairs of QTL reaching significance, testing for the individual significance of each of these four genotypic epistasis terms was done via individual $t$-tests.

Replication of experimentally derived QTL-Replication of significant experimentally identified QTL found was accomplished with HAM (also known as in silico analysis) using the Bayesian imputation-based association mapping approach (30). In this analysis, distance, duration, and speed data from 27 inbred strains of mice $[\mathrm{A} / \mathrm{J}, \mathrm{AKR} / \mathrm{J}, \mathrm{Balb} / \mathrm{cJ}, \mathrm{C} 3 \mathrm{H} / \mathrm{HeJ}$, C3Heb/FeJ, C57B/6J, C57L/J, CAST/EiJ, CBA/J, DBA/2J, MRL/MpJ, NZB/BinJ, SWR/J, and SPRET/EiJ (from Ref. 19); 129S1/SvImJ, C57Bl/10J, CE/J, LP/J, PL/J, SM/J, and WSB/ EiJ (Lightfoot, unpublished observations); and BTBR_T+_tf/J, DBA/1J, FVB/NJ, NOD/LtJ, RIIIS/J, and SJL/J (extrapolated from Ref. 29)] were associated with 1,272 SNPs derived from the Well-come Trust SNP database (36). The wild-type strains (CAST/EiJ, SPRET/EiJ, and $\mathrm{WSB} / \mathrm{EiJ}$ ) were included in this data set to increase the genomic diversity of the SNP-haplotype map (38). This data set averaged 636 SNPs on the two chromosomes analyzed with an average distance between each SNP of $181.8 \mathrm{kbp}(0.13 \mathrm{cM})$. Output from this analysis was a set of Bayesian factors calculated at each SNP site; $P$ values were calculated for each Bayesian factor on each chromosome, using 1,000 permutations. Significance of each SNP Bayesian factor was set a priori as 0.01 .

\section{RESULTS}

\section{Cohort Demographics and Activity Traits}

As expected, male mice were significantly $(P<0.05)$ heavier than female mice in all strains and substrains (Table 1). No difference was found between the four $F_{2}$ substrains in any of the activity traits (i.e., distance, duration, or speed), and thus these $F_{2}$ substrains were pooled for subsequent analyses. Weight and age did not exert significant effects on any of the activity traits and thus were dropped from further analyses. Strain $(P<0.005$, power $>0.85$ all traits $)$ and sex $(P<0.0001$, power $=0.99$ all traits $)$ were significant main effects in all traits, with post hoc analysis indicating that in general the $\mathrm{F}_{2}$ cohort had activity levels similar to the $\mathrm{C} 57 \mathrm{~L} /$ 
$\mathrm{J}$ progenitors, which were significantly higher than the $\mathrm{C} 3 \mathrm{H} / \mathrm{HeJ}$ progenitor strain (Figs. 1-3). Post hoc analysis indicated that the $\mathrm{C} 3 \mathrm{H} / \mathrm{HeJ}$ strain ran shorter distances than the other three groups (Fig. 1; $\sim 117 \%$ less on average than the $\mathrm{F}_{2}$ cohort) and ran less on a daily basis than the $\mathrm{C} 57 \mathrm{~L} / \mathrm{J}$ and $\mathrm{F}_{2}$ mice (Fig. $2 ; \mathrm{F}_{2}$ mice ran $\approx 81 \%$ longer than $\mathrm{C} 3 \mathrm{H} / \mathrm{HeJ}$ mice). Post hoc testing revealed that mice in all of the strains ran significantly different speeds from each other (Fig. 3; $\mathrm{C} 57 \mathrm{~L} / \mathrm{J}>\mathrm{F}_{1}>\mathrm{F}_{2}>\mathrm{C} 3 \mathrm{H} / \mathrm{HeJ}$ ). The female $\mathrm{F}_{2}$ mice exhibited significantly higher activity than the male $\mathrm{F}_{2}$ mice, running on average $47 \%$ farther, $39 \%$ longer, and $9 \%$ faster. Broadsense heritability $\left(\mathrm{d}^{2}\right)$ was high for all activity phenotypes: distance $\mathrm{d}^{2}=0.495 \pm 0.100$ (mean $\pm \mathrm{SE}$ ); duration $\mathrm{d}^{2}=0.586 \pm 0.82$; and speed $\mathrm{d}^{2}=0.469 \pm 0.105$.

\section{QTL Analyses}

Nongenetic adjustments and sex-specific QTL analysis-For the genetic analysis, the three activity traits were adjusted for sex, litter size, and rearing blocks to decrease trait variation and enhance the likelihood of identifying QTL. The standard deviation of distance, duration, and speed was reduced on average by $\sim 14 \%$ by the nongenetic variable adjustment. Normality tests showed that adjusted values for all three traits were normally distributed (data not shown). Not unexpectedly, all three traits were positively correlated $(P<0.01$; duration and distance, $r=0.92$; duration and speed, $r=0.45$; distance and speed, $r=0.71$ ). Given the sex differences we observed in the activity traits (Figs. 1-3), sex-specific QTL effects were tested. These tests indicated possible sex-QTL differences for distance and duration on chromosome 3 and for duration on chromosome 17. However, separate QTL analyses of the male and female cohorts for these three trait/chromosome combinations were not significant, showing no evidence of sex-specific QTL for any of the three activity traits. Therefore, all subsequent QTL analyses were based on the entire cohort without regard to sex.

One significant QTL (DUR13.1; Table 2) and three suggestive QTL were discovered for duration. These QTL contributed on average nearly $5 \%$ of the variation in duration, and a multiple regression model using $a$ and $d$ values from all four QTL showed that they jointly accounted for $14 \%$ (adjusted $R^{2}=11 \%$ ) of the total variance of duration. DUR13.1 exhibited a large positive additive genotypic value and a nonsignificant positive dominance value, suggesting that the $\mathrm{C} 57 \mathrm{~L} / \mathrm{J}$ allele increased duration.

At the 5\% experimentwise level, one significant distance QTL (DIST13.1) was discovered, colocalizing in a similar location as DUR13.1 - as did several of the suggestive distance and duration QTL - which probably represented pleiotropic QTL affecting both traits. The LPR score for DIST13.1 was 4.14, and this QTL contributed 6.36\% of the total variance in distance. Collectively, the significant and suggestive QTL for distance accounted for 21\% (adjusted $R^{2}$ value $\left.=18 \%\right)$ of the total variance of distance. DIST13.1 was similar to DUR13.1 in exhibiting significant positive genotypic values, indicating that the $\mathrm{C} 57 \mathrm{~L} / \mathrm{J}$ allele acts to increase distance.

Two QTL, SPD9.1 and SPD13.1, were significantly associated with speed of activity. $S P D 13.1$ probably represents the same QTL previously found for duration and distance (DIST13.1, DUR13.1), and SPD9.1 appears to colocalize with the suggestive QTL DIST9.1. The LPR score for SPD9.1 was 7.51, the highest achieved among the QTL for all three traits. The contributions of SPD9.1 and SPD13.1 were $11.27 \%$ and $6.10 \%$, respectively, and altogether the QTL for speed accounted for $34 \%$ (adjusted $R^{2}$ value $=30 \%$ ) of the total variance. Similar to the significant QTL for distance and duration, the positive additive values for SPD9.1 and SPD13.1 indicate that the C57L/J allele increased speed. 


\section{Epistasis Analyses}

Almost no evidence for epistasis among the detected QTL was found. $F$-tests for the pairwise interactions among QTL for duration and speed were all nonsignificant $(P>0.05)$. However, 1 of the 13 pairwise interactions among the QTL for distance, DIST5.1 with DIST9.1, was significant $(P=0.012)$. Of the four epistatic genotypic components associated with this interaction, $d d$ (standardized value $=-0.599)$ was significant $(P=0.009)$. This dominance by dominance epistasis suggests that the single-locus dominance genotypic value $(d)$ for DIST5. 1 changed depending on the genotype at DIST9.1 and vice versa. Inclusion of this epistatic term in the single-locus multiple regression model for distance increased the genetic contribution by $3 \%$ to a total variance of $24 \%$ (adjusted $R^{2}=0.21$ ).

\section{Haplotype Association Mapping}

We conducted HAM analyses for only chromosomes 9 and 13 since the QTL on these chromosomes had the greatest effects (Table 3$)$. Several significant $(P<0.01)$ HAM QTL were found within the confidence intervals of the significant experimental QTL we identified on chromosomes 9 (DIST9.1, SPD9.1) and 13 (DIST13.1, DUR13.1, SPD13.1). The significant HAM QTL colocalizing within the identified experimental QTL were not present when the C57L/J phenotype/genotype data were omitted from the HAM analysis (data not shown). Suggestive HAM QTL were also found for both DUR13.2 (rs3683883, $27.1 \mathrm{cM} ; P=0.15$ ), and DIST13.2 (rs13481817, $27.107 \mathrm{cM} ; P=0.021$ ), further refining the possible location of genes that play a role in regulating voluntary daily activity. The HAM analysis also identified 6 additional potential QTL linked to distance (5 on chromosome 9 and 1 on chromosome 13) and 12 additional potential QTL linked to speed (11 on chromosome 9 and 1 on chromosome 13). In particular, the areas surrounding the site at $35 \mathrm{cM}$ on chromosome 9 and $40 \mathrm{cM}$ on chromosome 13 were strongly linked with both distance and speed (Table 3), indicating the potential for further QTL not revealed in the present $\mathrm{F}_{2}$ cohort.

\section{DISCUSSION}

The results of this investigation, to our knowledge, represent the first identification of genomic locations associated with the regulation of voluntary wheel running activity in mice. In this study, we have also supported previous observations of the heritability of wheel running behavior and differential activity patterns between sexes and have noted a lack of differences between the $F_{2}$ substrains suggesting that the genetic influences on physical activity were not transferred predominantly through either the maternal or paternal lines. Furthermore, in the $\mathrm{F}_{2}$ cohort, we have identified two significant and several suggestive experimentally derived QTL that are linked with one or more of our three indexes of activity. The significant QTL on chromosomes 9 and 13 were confirmed with a HAM analysis, which also indicated several sites in these regions for further candidate gene investigation. Given the conserved synteny between human and mouse genomes, these results provide a significant foundation for further research investigating the genetic regulation of voluntary daily activity in rodents and humans (2).

It has only recently become accepted that voluntary daily activity has a significant heritable component $(12,16,19,31,35)$. In support of this contention, we found that the broad-sense heritability of wheel running behavior was substantial, ranging between $49 \%$ and $58 \%$ depending on the activity measurement considered, which is similar to previously reported values $(16,19)$. Not surprisingly, our broad-sense estimates of heritability are somewhat higher than previous non-adjusted narrow-sense heritability estimates for wheel running $(0.12-0.24$; Ref. 32); however, the additive genetic component estimates in the present study and previous studies (adjusted realized heritability $=0.28$; Ref. 32) are similar. 
Whereas the portion of the heritability/phenotypic variance that was accounted for by the QTL ranged from $11 \%$ to $34 \%$ depending on the activity index used, it is probable that there are other QTL or genetic factors that explain more of the variability that were not uncovered in our limited cohort of $F_{2}$ animals. For example, Tsao et al. (34) demonstrated that an overexpression of GLUT4 glucose transporters leads to a fourfold increase in daily activity in male mice. However, the GLUT4 gene is located on chromosome $11(40 \mathrm{cM})$, an area in which none of the QTL we identified in the present study was found. Our haplotype mapping results certainly indicated other sites on chromosomes 9 and 13 that may contain QTL that influence activity. Additionally, while the near absence of significant epistasis among the identified QTL was somewhat surprising $(37,39)$, this does not rule out the possibility of epistasis among loci that were not detected as main-effect QTL. While these possibilities will require further investigation to identify all of the chromosomal locations that are linked to the regulation of physical activity, the present study has identified two major and several other suggestive loci that control voluntary daily activity in mice.

As additional QTL for measures of voluntary daily activity are eventually discovered in other mouse models, it will be interesting to see whether their mode of action is comparable to that seen for the QTL we have located. For example, it is unclear whether the lack of sex-specific QTL for the activity traits measured here indicates that the sex-mediated regulation of activity is a result of other biological factors not related to genetic regulation (i.e., "downstream" of genetic regulation) or whether we simply did not have sufficient power to detect them in our mouse population (see below). Certainly QTL that act differentially in the sexes have been detected for other traits in mice $(14,21)$. The mean levels of the standardized additive/ dominance genotypic values and percentage contributions for our QTL, however, are generally quite comparable to those reported by Kenney-Hunt et al. (11) for a large number of QTL influencing a battery of body size components in a $\mathrm{LG} / \mathrm{J} \times \mathrm{SM} / \mathrm{J}$ intercross population of mice.

While no other study has identified QTL directly linked to the activity traits we used (i.e., wheel running activity), other studies have identified QTL linked to other locomotion-related behaviors. In 25 recombinant inbred (RI) mouse strains developed from C57BL/6J and DBA/ 2J inbred strains, Phillips et al. (25) identified five QTL in 87-day-old female mice associated with the magnitude of horizontal movement in an activity monitoring chamber. Of these five QTL, three (chromosome 9, 26-36 cM; chromosome 13, 9-10 cM; chromosome 5, 20-30 cM) colocalize with QTL identified in the present study-the significant QTL we identified on chromosomes 9 (DIST9.1 and SPD9.1) and 13 (DUR13.1, DIST13.1, and SPD13.1) as well as the suggestive QTL SPD5.1. Unfortunately, Phillips and colleagues only reported measures of correlation and did not report any measure of QTL strength; thus it is unknown whether these QTL reached either an experimentwise or a chromosomewise level of significance (25).

Using 22 recombinant inbred rat strains- the HXB/BXH RI strains derived from SHR/O1a and the inbred congenic BN.Lx/Cub strain-that were 11-13 wk old, Conti et al. (5) identified two significant QTL on chromosomes 3 (47 cM; D3Rat180 proximal marker) and 18 (40 cM; D18Rat55 proximal marker) related to open-maze locomotion behavior. Additionally, Gershenfeld and colleagues (8) investigated the genetic regulation of open-field behavior (e.g., vertical rearing and response to novelty) and identified several significant QTL on chromosomes 1,3,10, and 19 and five additional suggestive QTL in 10- to 11-wk-old $\mathrm{F}_{2}$ mice derived from $\mathrm{A} / \mathrm{J}$ and $\mathrm{C} 57 \mathrm{~B} 1 / 6 \mathrm{~J}$ progenitor strains. Comparisons of the results from Conti et al. (5) and Gershenfeld et al. (8) to those in the present study are difficult because of the shorter time interval of the locomotor phenotyping used by Conti et al. (5-min observation) and Gershenfeld et al. (15-min observation) compared with our longer-term measurements (21 days). Additionally, Mill et al. (23) observed that home cage activity in mice, similar to our measures of activity, was not correlated with open-field locomotor testing measures similar to those employed by Gershenfeld et al. (8). Furthermore, open-field testing and maze testing 
similar to those used by Conti et al. (5) are now widely considered a measurement of fear and anxiety rather than voluntary locomotion in rodents (24). Thus it is not surprising that none of the QTL (or QTL homologs) identified by either Conti and colleagues (5) or Gershenfeld et al. (8) is similar to the QTL associated with the longer-term indexes of activity that we used in this study.

Additionally, other locomotor-associated behaviors have been examined genetically, such as the efforts made to determine QTL associated with the amplitude of the daily oscillation in locomotion between day and night (33). This effort monitored activity with a tethered-EMG implant system in C57BL/6, Balb/cBy, and 13 RI strains. Unfortunately, only male mice were monitored for a 48-h period and no significant QTL were discovered associated with the variation in activity (total 24-h activity among all of the strains was not different). However, several suggestive QTL were identified by these authors, one being located on chromosome $12(23 \mathrm{cM})$, in a location similar to our SPD12.1.

It is tempting to hypothesize that QTL for physiological traits associated with high exercise endurance (e.g., mitochondrial density, cardiac output) are associated with the functional ability to be active for long periods of time. However, these hypotheses are not justified at this time because of two issues. First, several studies, including work from our lab, have shown that physical activity and exercise endurance in mice are not correlated and are therefore probably distinct phenotypes (e.g., Refs. 16,19,20). Further strengthening the contention that maximal exercise endurance and high/low physical activity are distinct phenotypes is the fact that the activity QTL noted in the present study do not colocalize with any of the mouse QTL recently published for exercise endurance (21). Second, given our inability to determine the length and intensity of the individual exercise bouts that the mice were performing, it is difficult to determine what type of physiological trait might have functionally allowed the increased activity in our $\mathrm{F}_{2}$ cohort. If the mice were performing multiple short, intense bouts of exercise, we would naturally want to investigate colocalization of QTL of physiological traits favoring intense exercise bouts (e.g., \% of type II fiber composition, increased lactate dehydrogenase). Conversely, if the mice were completing exercise in longer, less intensive bouts, then we would be interested in QTL for traits leading to longer duration exercise (e.g., type I fiber composition, increased mitochondrial biogenesis). These types of QTL comparisons are logical for future studies when the types of activity bouts can be monitored.

Our general finding of higher activity levels in female mice supports results from multiple rodent studies that have found similar results (e.g., Refs. 12,17,19). However, as noted above, the lack of sex-related QTL could indicate that the differential regulation of activity due to sex is downstream of any genetic regulation. In fact, studies have clearly established that estrogen mediates physical activity through the estrogen receptor- $\alpha$ pathway (24). While the subsequent downstream pathways activated by the estrogen receptor- $\alpha$ pathway are still somewhat unclear, it has been postulated that estrogen receptor- $\alpha$ modulates several neurotransmitters, including dopamine in the female, which may lead to increased physical activity (24). Supporting a possible dopamine linkage are two studies $(26,27)$ that have suggested a role for various dopamine mechanisms in influencing activity. Interestingly, while no genes with known control of any sex hormones are located in the QTL regions identified in the present study, identified QTL that affect the physiological behavior of dopamine colocalize within some of the suggestive QTL sites we identified. $D b h$ (chromosome $2,15.5 \mathrm{cM}$ ), a gene that produces dopamine $\beta$-hydroxylase, an enzyme that catalyzes the dopamine-to-norepinephrine pathway, is located within our SPD2.1 QTL.

Additionally, while no dopamine receptor genes are located within any of our identified significant QTL, there are two QTL, Drb2 (chromosome 5, $54.0 \mathrm{cM}$ ) and Drb5 (chromosome $12,25.0 \mathrm{cM}$ ), both of which are associated with dopamine receptor binding, that colocalize 
within our identified SPD5.1 and SPD12.1 sites. Therefore, given the location of these dopamine-associated regions within three of our suggestive QTL sites and the apparent lack of estrogen-controlling genes in any of the identified QTL sites, we hypothesize that while the genetic regulation of activity may involve dopamine (i.e., through dopamine receptor and/or dopamine metabolism) the sex-related estrogenic effects on activity appear to be nongenetic in nature and potentially occur downstream of other genetic regulatory mechanisms.

In summary, this study has experimentally identified 4 significant and at least 14 suggestive QTL associated with spontaneous activity in mice. The significant QTL on chromosomes 9 and 13 were validated with a HAM approach, which also identified several other genomic loci where potential QTL may exist. This study also noted a clear sex difference in activity patterns, but we hypothesize that this sex difference results from a nongenetic mechanism functioning downstream of genetic regulation. Future research will focus on reducing the intervals where these identified QTL exist to ultimately identify genes that regulate physical activity.

\section{ACKNOWLEDGMENTS}

The authors thank Jessica Moser, Sarah Carter, Matt Yost, Anna Vordermark, Amy Kleinfehn-Knab, Robert Bowen, Felicia Dangerfield-Persky, Sean Courtney, and Alicia Trynor for their technical expertise and the Vivarium staff for their animal husbandry skills.

\section{REFERENCES}

1. Beavis, WD. The power and deceit of QTL experiments: lessons from comparative QTL studies.. Corn and Sorghum Research Conference.; Washington, DC. American Seed Trace Association; 1994. p. 252-268.

2. Bogue MA. Mouse Phenome Project: understanding human biology through mouse genetics and genomics. J Appl Physiol 2003;95:1335-1337. [PubMed: 12970372]

3. Chakravarthy M, Booth F. Eating, exercise, and "thrift" genotypes: connecting the dots toward an evolutionary understanding of modern chronic diseases. J Appl Physiol 2004;96:3-10. [PubMed: 14660491]

4. Cheverud J. A simple correction for multiple comparisons in interval mapping genome scans. Heredity 2001;87:52-58. [PubMed: 11678987]

5. Conti LH, Jirout M, Breen L, Vanella JJ, Schork NJ, Printz MP. Identification of quantitative trait loci for anxiety and locomotion phenotypes in rat recombinant inbred strains. Behav Genet 2004;34:93103. [PubMed: 14739700]

6. Eppig J, Bult C, Kadin J, Richardson J, Blake J, members of the Mouse Genome Database Group. The Mouse Genome Database (MGD): from genes to mice - a community resource for mouse biology. Nucleic Acids Res 2005;33:D471-D475. [PubMed: 15608240]

7. Festing, MFW. Inbred Strains in Biomedical Research. Oxford Univ. Press; New York: 1979. Notes on genetic analysis.; p. 80-98.

8. Gershenfeld HK, Neumann PE, Mathis C, Crawley JN, Li Z, Paul SM. Mapping quantitative trait loci for open-field behavior in mice. Behav Genet 1997;27:201-210. [PubMed: 9210791]

9. Goodman S. Multiple comparisons, explained. Am J Epidemiol 1998;147:807-812. [PubMed: 9583709]

10. Haley CS, Knott SA. A simple regression technique for mapping quantitative trait loci in line crosses using flanking markers. Heredity 1992;69:315-324. [PubMed: 16718932]

11. Kenney-Hunt J, Vaughn T, Pletscher L, Peripato A, Routman E, Cothran K, Durand D, Norgard E, Perel C, Cheverud J. Quantitative trait loci for body size components in mice. Mamm Genome 2006;17:526-537. [PubMed: 16783635]

12. Koteja P, Swallow JG, Carter PA, Garland T Jr. Energy cost of wheel running in house mice: implications for coadaptation of locomotion and energy budgets. Physiol Biochem Zool 1999;72:238-249. [PubMed: 10068627] 
13. Lander ES, Botstein D. Mapping mendelian factors underlying quantitative traits using RFLP linkage maps. Genetics 1989;121:185-199. [PubMed: 2563713]

14. Leamy LJ, Pomp D, Eisen EJ, Cheverud JM. Pleiotropy of quantitative trait loci for organ weights and limb bone lengths in mice. Physiol Genomics 2002;10:21-29. [PubMed: 12118102]

15. Leamy LJ, Routman EJ, Cheverud JM. Quantitative trait loci for early- and late-developing skull characters in mice. Am Naturalist 1999;153:201-214.

16. Lerman I, Harrison BC, Freeman K, Hewett TE, Allen DL, Robbins J, Leinwand LA. Genetic variability in forced and voluntary endurance exercise performance in seven inbred mouse strains. $\mathrm{J}$ Appl Physiol 2002;92:2245-2255. [PubMed: 12015333]

17. Li JS, Huang YC. Early androgen treatment influences the pattern and amount of locomotion activity differently and sexually differentially in an animal model of ADHD. Behav Brain Res 2006;175:176182. [PubMed: 16979765]

18. Li X, Quigg RJ, Zhou J, Xu S, Masinde G, Mohan S, Baylink DJ. A critical evaluation of the effect of population size and phenotypic measurement on QTL detection and localization using a large F2 murine mapping population. Genet Mol Biol 2006;29:166-173.

19. Lightfoot JT, Turner MJ, Daves M, Vordermark A, Kleeberger SR. Genetic influence on daily wheel running activity level. Physiol Genomics 2004;19:270-276. [PubMed: 15383638]

20. Lightfoot JT, Turner MJ, DeBate KA, Kleeberger SR. Interstrain variation in murine aerobic capacity. Med Sci Sports Exerc 2001;33:2053-2057. [PubMed: 11740298]

21. Lightfoot JT, Turner MJ, Knab AK, Jedlicka AE, Oshimura T, Marzec JM, Gladwell W, Leamy L, Kleeberger S. Quantitative trait loci associated with maximal exercise endurance in mice. J Appl Physiol 2007;103:105-110. [PubMed: 17412788]

22. Lynch, M.; Walsh, B. Genetics and Analysis of Quantitative Traits. Sinauer; Sunderland, MA: 1998.

23. Mill J, Galsworthy M, Paya-Cano J, Sluyter F, Schalkwyk L, Plomin R, Asherson P. Home-cage activity in heterogeneous stock (HS) mice as a model of baseline activity. Genes Brain Behav 2002;1:166-173. [PubMed: 12884972]

24. Morgan M, Schulkin J, Pfaff D. Estrogens and non-reproductive behaviors related to activity and fear. Neurosci Biobehav Rev 2004;28:55-63. [PubMed: 15036933]

25. Phillips TJ, Huson MG, McKinnon CS. Localization of genes mediating acute and sensitized locomotor responses to cocaine in BSD/Ty recombinant inbred mice. J Neurosci 1998;18:3023-3034. [PubMed: 9526019]

26. Rhodes JS, Garland T Jr. Differential sensitivity to acute administration of Ritalin, apomorphine, SCH 23390, but not raclopride in mice selectively bred for hyperactive wheel-running behavior. Psychopharmacology (Berl) 2003;167:242-250. [PubMed: 12669177]

27. Rhodes JS, Hosack G, Girard I, Kelley A, Mitchell G, Garland T Jr. Differential sensitivity to acute administration of cocaine, GBR 12909, and fluoxetine in mice selectively bred for hyperactive wheelrunning behavior. Psychopharmacology (Berl) 2001;158:120-131. [PubMed: 11702085]

28. Roberts C, Barnard R. Effects of exercise and diet on chronic disease. J Appl Physiol 2005;98:3-30. [PubMed: 15591300]

29. Seburn, K. Mouse Phenome Database: metabolic characterization. Jackson Laboratory; 2001.

30. Servin B, Stephens M. Efficient multipoint analysis of association studies: candidate regions and quantitative traits. PLOS Genet 2007;3:e114. [PubMed: 17676998]

31. Stubbe JH, Boomsma DI, De Geus EJC. Sports participation during adolescence: a shift from environmental to genetic factors. Med Sci Sports Exerc 2005;37:563-570. [PubMed: 15809553]

32. Swallow JG, Carter PA, Garland T Jr. Artificial selection for increased wheel-running behavior in house mice. Behav Genet 1998;28:227-237. [PubMed: 9670598]

33. Toth LA, Williams RW. A quantitative genetic analysis of locomotor activity in CXB recombinant inbred mice. Behav Genet 1999;29:319-328. [PubMed: 10765560]

34. Tsao TS, Li J, Change KS, Stenbit AE, Galuska D, Anderson JE, Zierath JR, McCarter RJ, Charron MJ. Metabolic adaptations in skeletal muscle overexpressing GLUT4: effects on muscle and physical activity. FASEB J 2001;15:958-969. [PubMed: 11292656]

35. Turner MJ, Kleeberger SR, Lightfoot JT. Influence of genetic background on daily running-wheel activity differs with aging. Physiol Genomics 2005;19:270-276. [PubMed: 15383638] 
36. Wellcome Trust Centre for Human Genetics. Wellcome-CTC Mouse Strain SNP Genotype Set. 2005.

37. Wolf J, Pomp D, Eisen E, Leamy L. The contribution of epistatic pleiotropy to the genetic architecture of covariation among polygenic traits in mice. Evol Dev 2006;8:468-476. [PubMed: 16925682]

38. Yang H, Bell TA, Churchill GA, Pardo-Manual de Villena F. On the subspecific origin of the laboratory mouse. Nat Genet 2007;39:1100-1107. [PubMed: 17660819]

39. Yi N, Yandell B, Churchill GA, Allison D, Eisen E, Pomp D. Bayesian model selection for genomewide epistatic quantitative trait loci analysis. Genetics 2005;170:1333-1344. [PubMed: 15911579] 


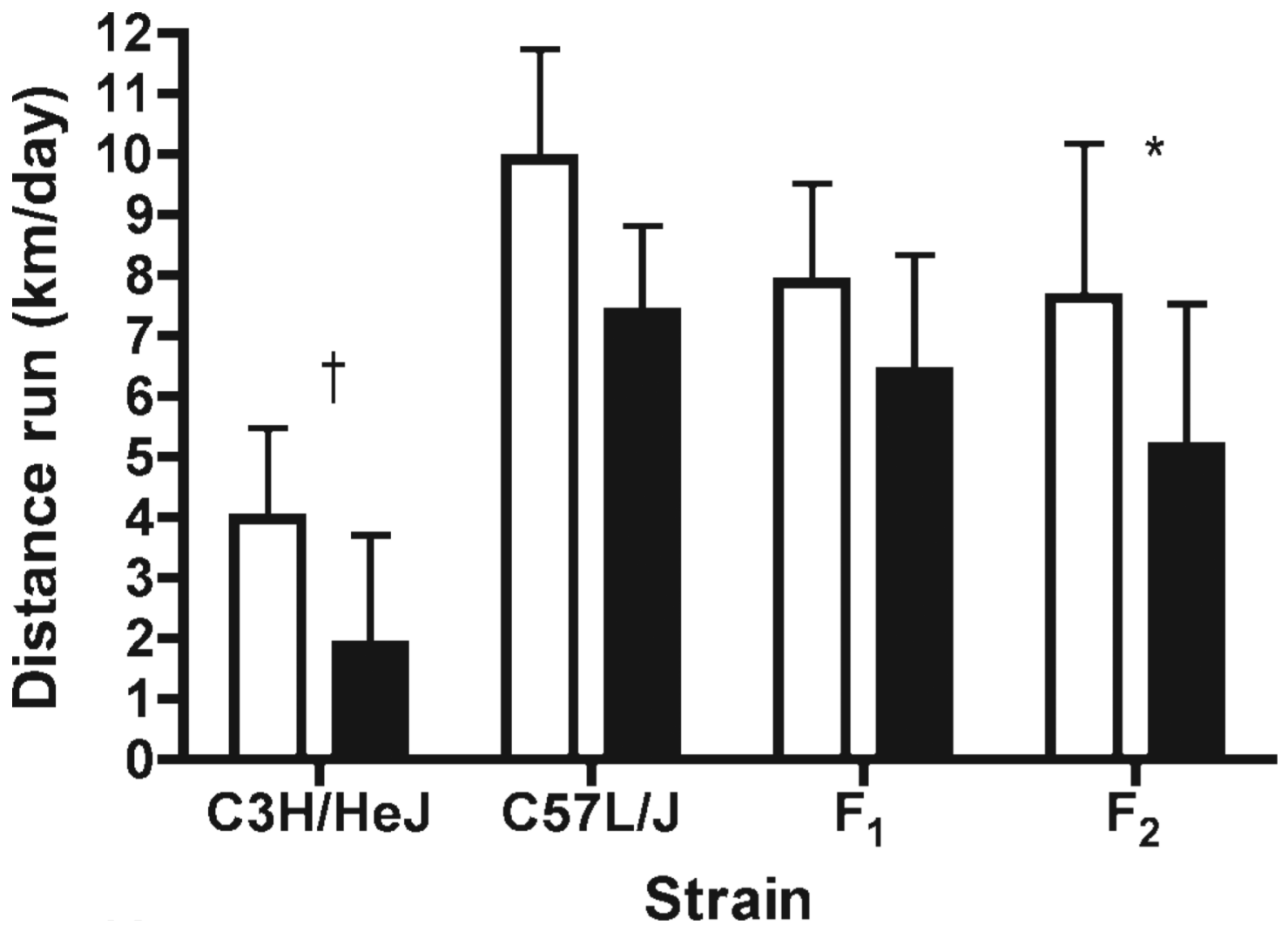

Fig. 1.

Daily distance run by sex and strain (means \pm SD). Open bars, females; filled bars, males. *Significantly different between sexes within strain; $†$ significantly less distance than other strains. 


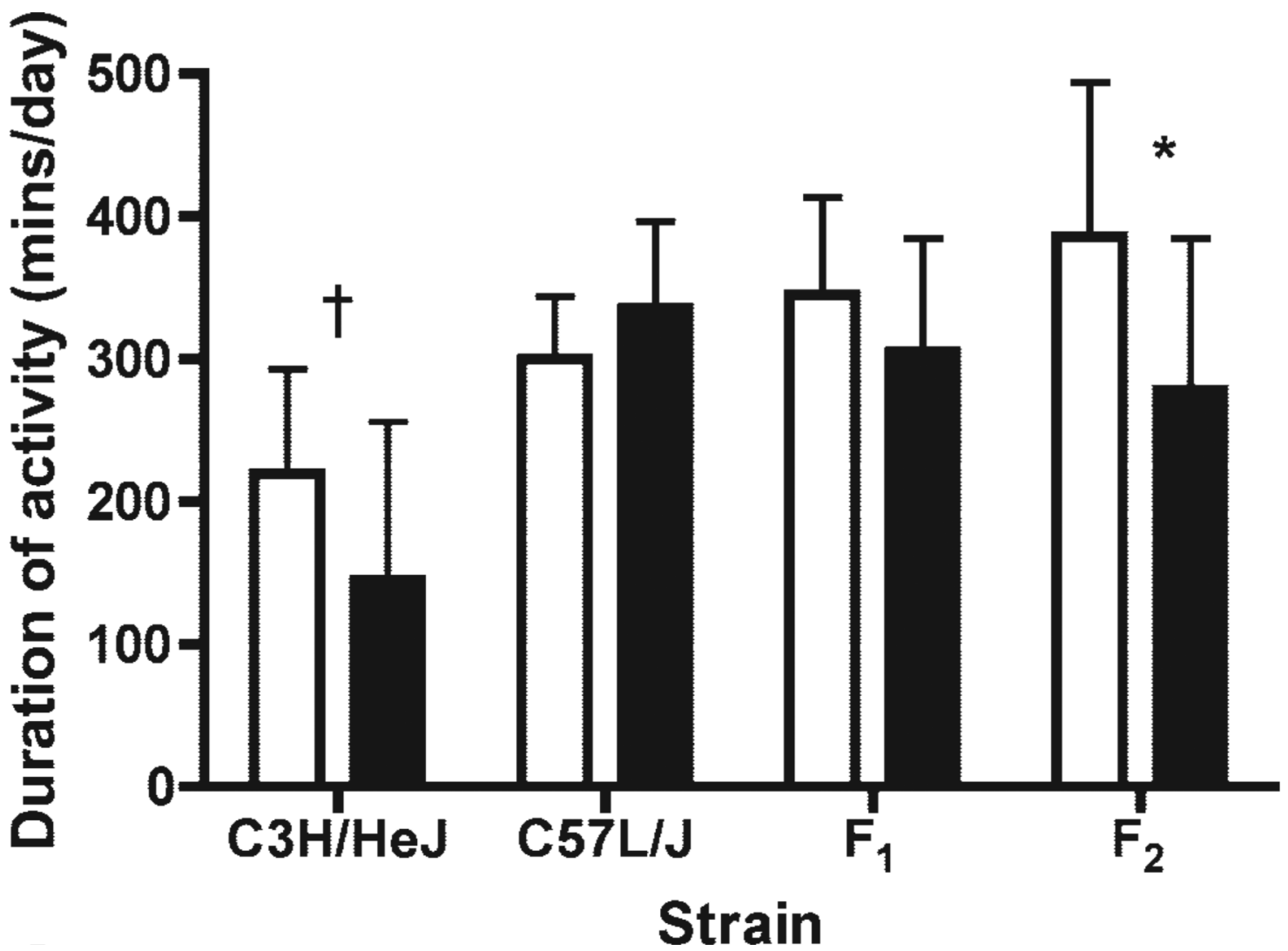

Fig. 2.

Duration of activity by sex and strain (means \pm SD). Open bars, females; filled bars, males. *Significantly different between sexes within strain; † significantly less duration than C57L/J and $\mathrm{F}_{2}$ mice. 


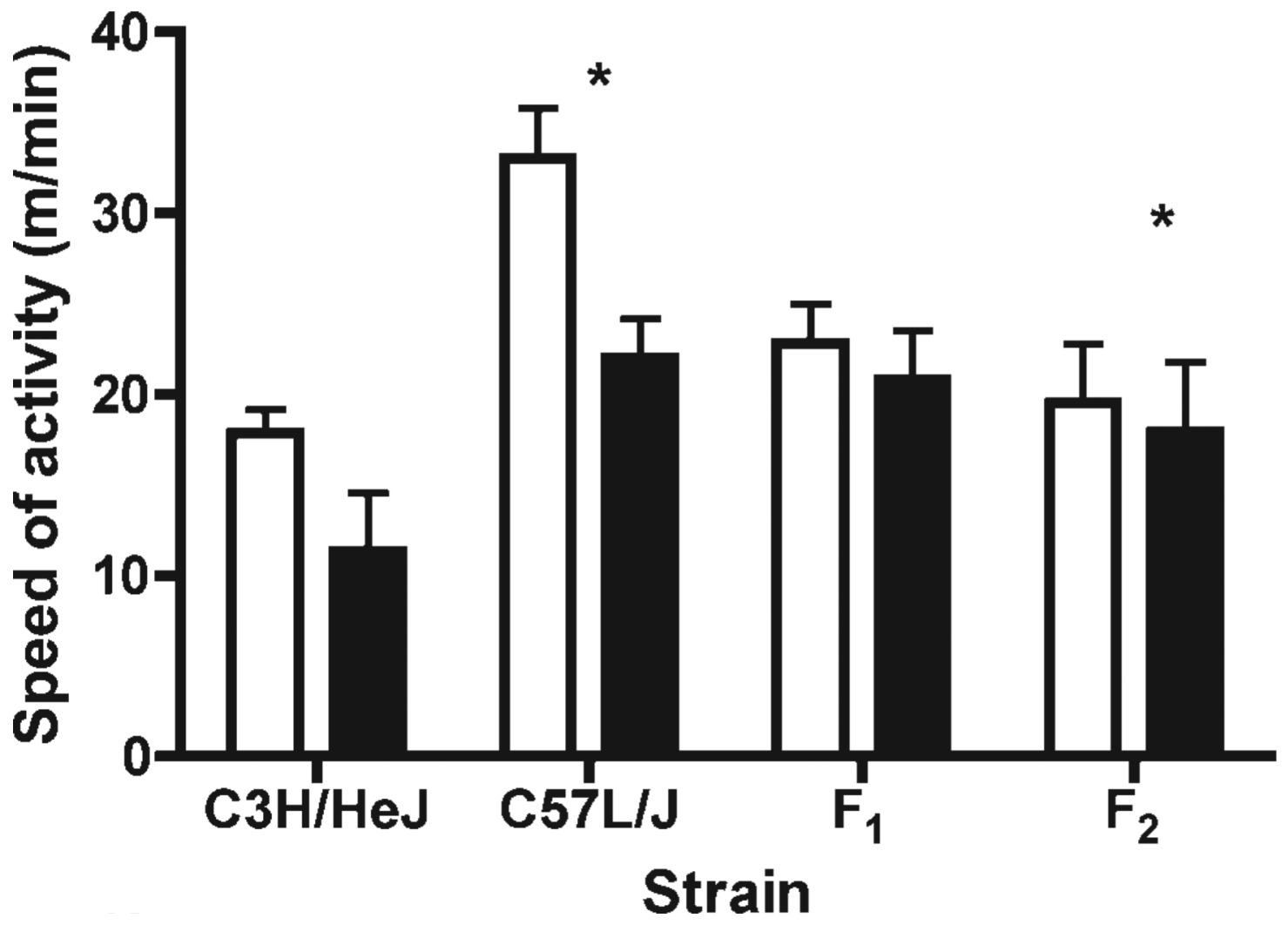

Fig. 3.

Average speed of activity by sex and strain (means $\pm \mathrm{SD}$ ). Open bars, females; filled bars, males. *Significantly different between sexes within strain. All strains ran at speeds significantly different from each other. 


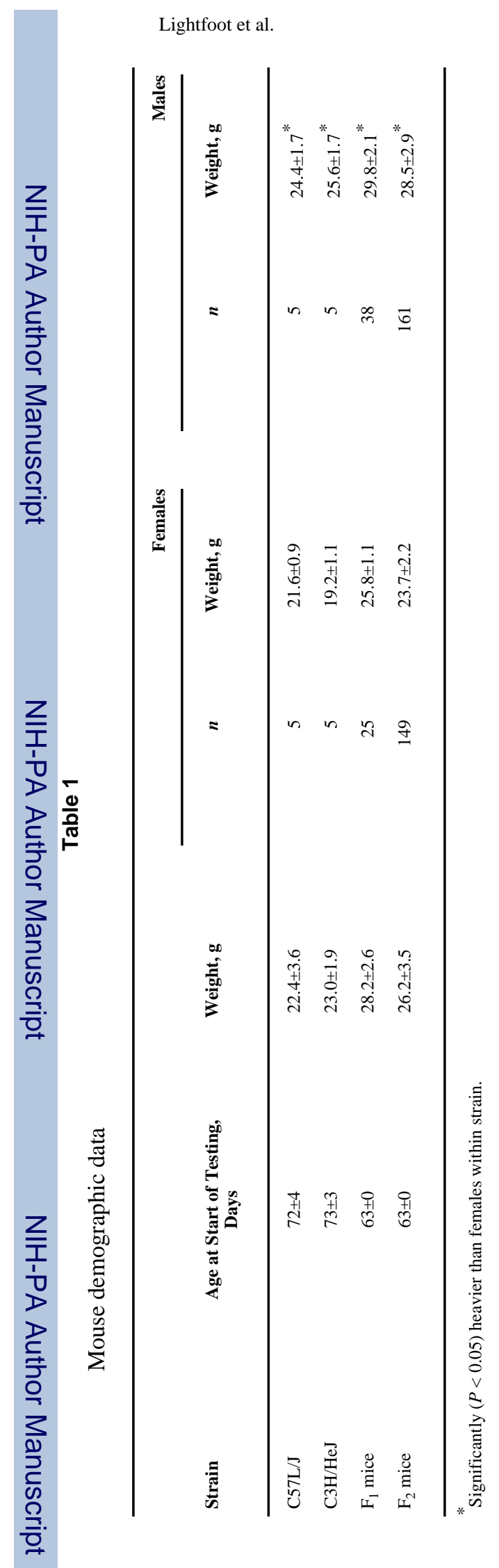

Physiol Genomics. Author manuscript; available in PMC 2009 September 16. 


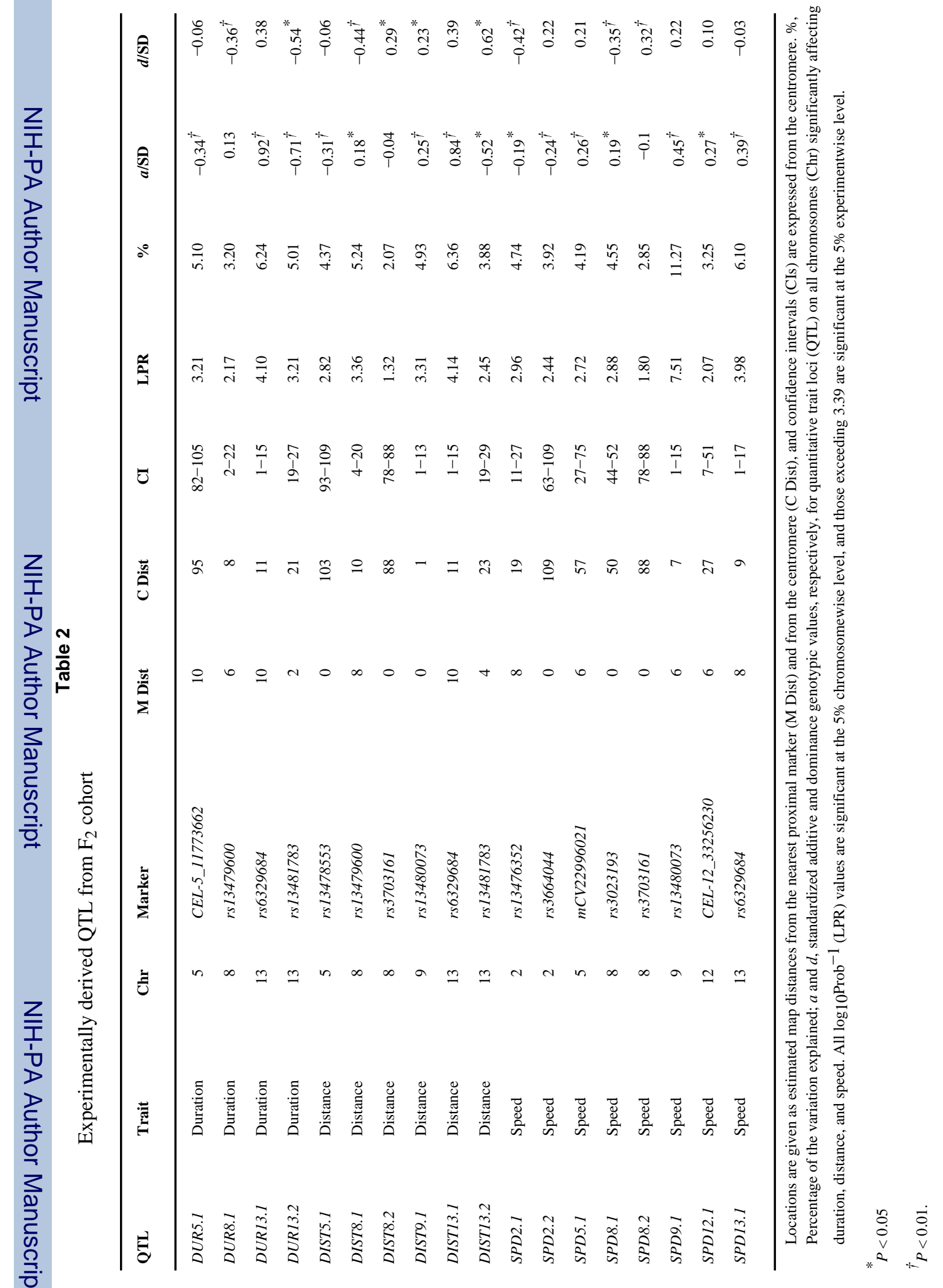




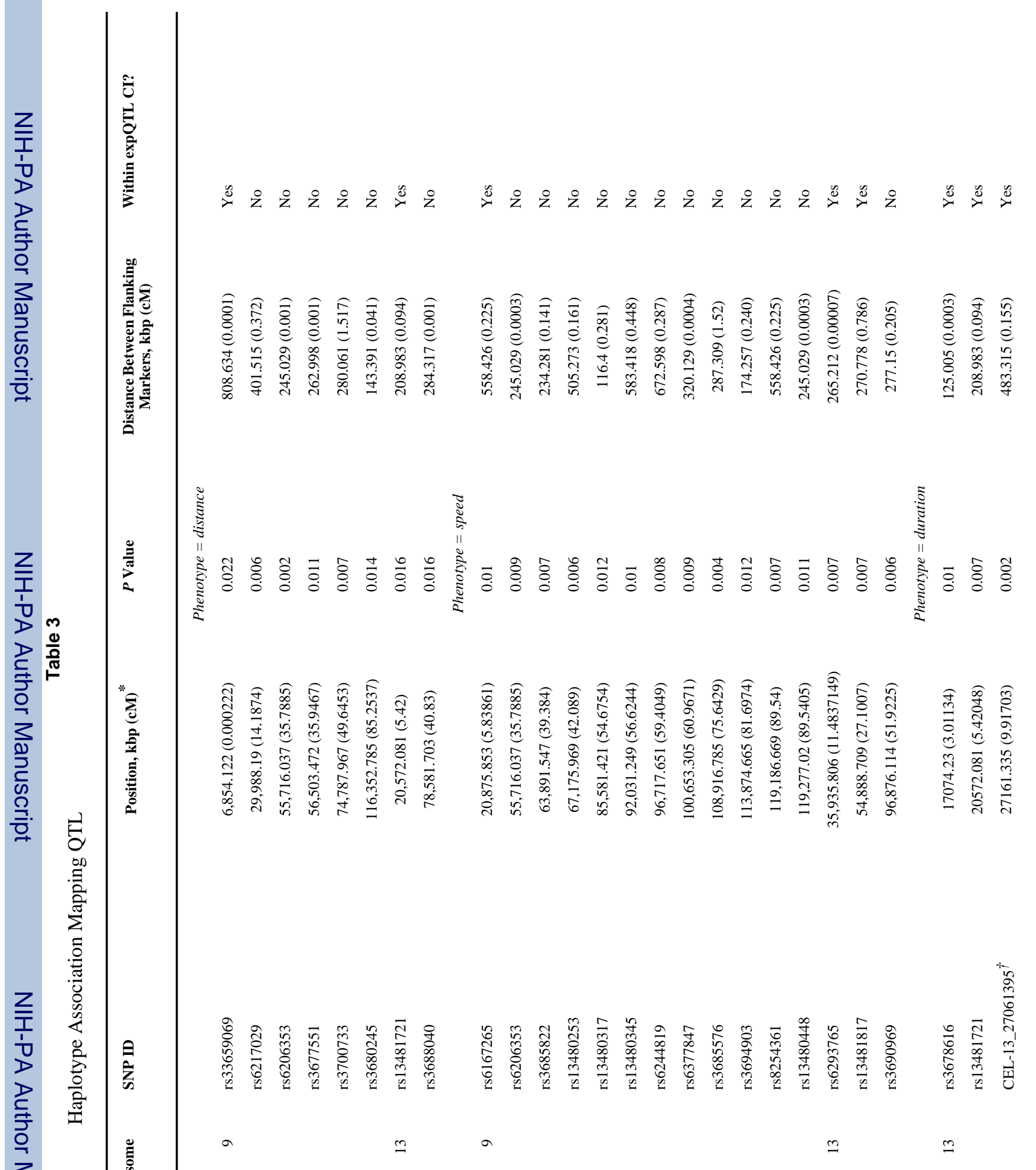




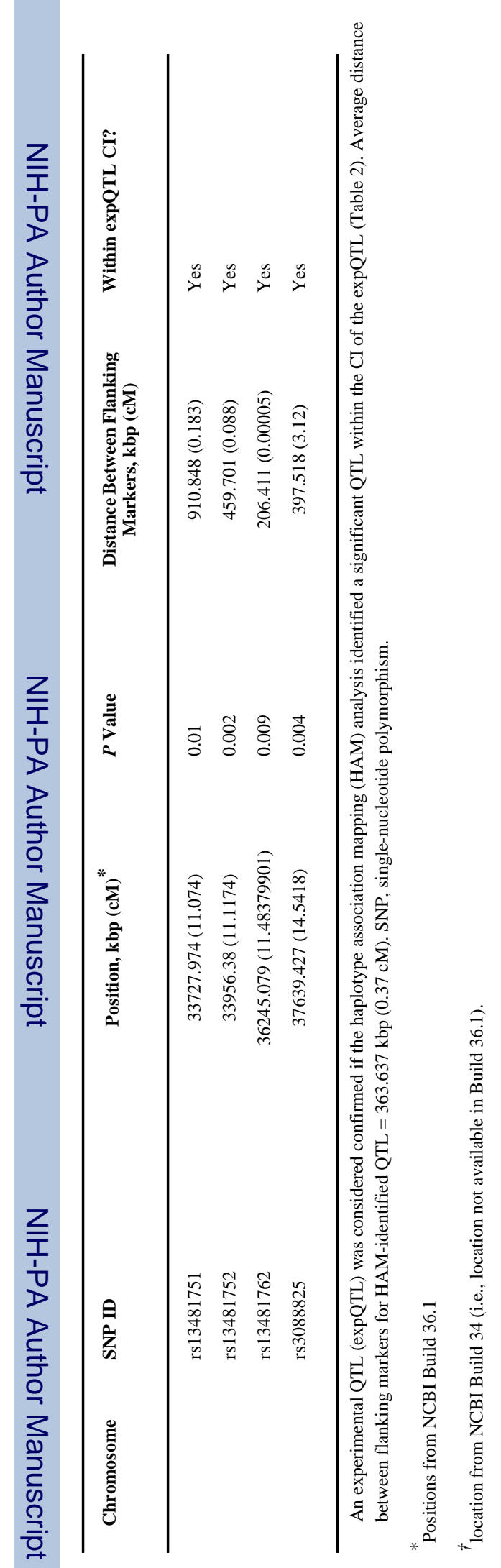

Physiol Genomics. Author manuscript; available in PMC 2009 September 16. 